J. Nonlinear Var. Anal. 3 (2019), No. 2, pp. 141-148

Available online at http://jnva.biemdas.com

https://doi.org/10.23952/jnva.3.2019.2.03

\title{
LOCAL FIXED POINT RESULTS FOR GRAPHIC CONTRACTIONS
}

\author{
ADRIAN PETRUŞEL ${ }^{1,2}$ \\ ${ }^{1}$ Department of Mathematics, Babeş-Bolyai University, Cluj-Napoca, Romania \\ ${ }^{2}$ Academy of Romanian Scientists, Bucharest, Romania
}

\begin{abstract}
The aim of this paper is to present some local fixed point results for graphic contractions in complete metric spaces. Keywords. Graphic contraction; Graph of a mapping; Complete metric space; Fixed point; Local fixed point theorem.
\end{abstract}

2010 Mathematics Subject Classification. 47H10, 54H25.

\section{INTRODUCTION}

Let $X$ be a nonempty set and let $f: X \rightarrow X$ be an operator. A solution of the fixed point equation

$$
x=f(x), x \in X
$$

is called a fixed point of $f$. We denote by Fix $(f)$ the fixed point set of $f$, i.e., Fix $(f):=\{x \in X \mid f(x)=x\}$ and by $\operatorname{Graph}(f):=\{(x, f(x)): x \in X\}$ the graph of operator $f$. The symbol $I(f)$ denotes the set of all invariant subsets of $f$, i.e., $I(f):=\{Y \subset X: f(Y) \subset Y\}$.

If $(X, d)$ is a metric space, then, by definition, $f$ is a weakly Picard operator if

$$
f^{n}(x) \rightarrow x^{*}(x) \in F i x(f) \text { as } n \rightarrow \infty, \text { for all } x \in X .
$$

A weakly Picard operator having a unique fixed point is a Picard operator.

For example, if $(X, d)$ is a complete metric space and $f: X \rightarrow X$ is a contraction mapping, i.e, there exists $\alpha \in] 0,1[$ such that

$$
d(f(x), f(y)) \leq \alpha d(x, y) \text {, for every } x, y \in X,
$$

then $f$ is a Picard operator. If the above condition is imposed only for pairs $(x, y) \in \operatorname{Graph}(f)$, then the mapping is called a graphic contraction. A continuous graphic contraction (or, more generally, a graphic contraction with a closed graph) on a complete metric space is a weakly Picard operator.

The purpose of this paper is to present some local fixed point results in complete metric spaces for graphic contractions.

E-mail address: petrusel@math.ubbcluj.ro.

Received April 14, 2018; Accepted March 11, 2019.

(C)2019 Journal of Nonlinear and Variational Analysis 


\section{MAIN RESULTS}

Let $(X, d)$ be a complete metric space and let $f: X \rightarrow X$ be a weakly Picard operator. Then, we denote by $f^{\infty}(x) \in F i x(f)$ the limit of the sequence $\left(f^{n}(x)\right)_{n \in \mathbb{N}}$ of successive approximations for $f$ starting from $x \in X$.

A weakly Picard operator $f: X \rightarrow X$ for which there exists a function $\psi: \mathbb{R}_{+} \rightarrow \mathbb{R}_{+}$increasing, continuous in 0 and satisfying $\psi(0)=0$, such that

$$
d\left(x, f^{\infty}(x)\right) \leq \psi(d(x, f(x)), \text { for all } x \in X,
$$

is called a weakly $\psi$-Picard operator. For example, a graphic contraction with constant $\alpha \in] 0,1[$ is a weakly $\psi$-Picard operator with $\psi(t):=\frac{1}{1-\alpha} t$.

Moreover, a Picard operator, with its unique fixed point denoted by $x^{*} \in X$, for which there exists a function $\psi: \mathbb{R}_{+} \rightarrow \mathbb{R}_{+}$increasing, continuous in 0 and satisfying $\psi(0)=0$, such that

$$
d\left(x, x^{*}\right) \leq \psi(d(x, f(x)), \text { for all } x \in X,
$$

is called a $\psi$-Picard operator. For example, an $\alpha$-contraction is a $\psi$-Picard operator with $\psi(t):=\frac{1}{1-\alpha} t$.

For more results on the weakly Picard operator theory, we refer the reader to $[1,12,13,14]$ and the references therein

Definition 2.1. Let $(X, d)$ be a metric space and let $f: X \rightarrow X$ be an operator. Then, $f$ is called:

(i) an $\alpha$-contraction if $\alpha \in] 0,1[$ and

$$
d(f(x), f(y)) \leq \alpha d(x, y) \text {, for every } x, y \in X .
$$

(ii) a graphic $\alpha$-contraction if $\alpha \in] 0,1[$ and

$$
d\left(f(x), f^{2}(x)\right) \leq \alpha d(x, f(x)), \text { for every } x \in X .
$$

Any $\alpha$-contraction is a graphic $\alpha$-contraction, but the inverse may not be true.

Example 2.1. Let $X:=[0,1] \cup[2,3]$ and let $f: X \rightarrow X$ be defined by

$$
f(x):= \begin{cases}\frac{1}{2} x, & x \in[0,1], \\ \frac{1}{2} x+\frac{3}{2}, & x \in[2,3] .\end{cases}
$$

Then, $f$ is a $\frac{1}{2}$-contraction on $[0,1]$ and on $[2,3]$ and $F i x(f)=\{0,3\}$. Moreover, $f$ is a continuous graphic $\frac{1}{2}$-contraction, but it is not a contraction.

In 1972, Rus proved in [11] (see also [14]) that a graphic contraction on a complete metric space has at least one fixed point provided that its graph is closed.

Another fixed point result for graphic contractions in the framework of a Banach space was given by Subrahmanyam in [16]. In his paper, Subrahmanyam introduced the concept of the Banach operator of type $\alpha$ to designate a graphic $\alpha$-contraction in the sense of Definition 2.1. His result (see Corollary 2 in [16]) says that if $S$ is a closed subset of a Banach space and $f: S \rightarrow S$ is a continuous Banach operator of type $\alpha$, then it has a fixed point.

By the following example, we notice the importance of the continuity condition on $f$ (or, more generally, of the closed graph condition), see also [2] and the references therein. 
Example 2.2. Let $X:=[0,1]$ and let $f: X \rightarrow X$ be defined by

$$
f(x):= \begin{cases}\frac{1}{2}, & x=0, \\ \frac{1}{2} x, & x \in] 0,1] .\end{cases}
$$

Then, $f$ is a graphic $\frac{1}{2}$-contraction on $[0,1]$ with $\operatorname{Fix}(f)=\emptyset$. Moreover, for every $x \in X,\left(f^{n}(x)\right)_{n \in \mathbb{N}}$ converges to 0 as $n \rightarrow \infty$. Notice that $f$ is discontinuous and $\operatorname{Graph}(f)$ is not a closed set.

An interesting fixed point result (in complete metric spaces) for operators $f: X \rightarrow X$ satisfying the graphic contraction condition on the orbit of $f$ at a given point $x \in X$ was proved by Hicks and Rhoades in [3]. For a recent existence and uniqueness theorem for graphic contractions in complete metric spaces, readers are referred to [2].

A new research direction in fixed point theory was initiated by Ran and Reurings in [10] via the following result.

Theorem 2.1. Let $X$ be a nonempty set endowed with a partial order relation " $\preceq$ ” and let $d: X \times X \rightarrow$ $\mathbb{R}_{+}$be a complete metric on $X$. Let $f: X \rightarrow X$ be an operator which is continuous with respect to $d$ and increasing with respect to " $\preceq$ ”. Suppose that there exist a constant $\alpha \in] 0,1\left[\right.$ and an element $x_{0} \in X$ such that:

(i) $d(f(x), f(y)) \leq \alpha d(x, y)$, for all $x, y \in X$ with $x \preceq y$.

(ii) $x_{0} \preceq f\left(x_{0}\right)$.

Then Fix $(f) \neq \emptyset$ and the sequence of successive approximations $\left(f^{n}(x)\right)_{n \in \mathbb{N}}$ starting from any point $x \in X$, which is comparable to $x_{0}$, converges to a fixed point of $f$.

For some extensions of the above result, see $[5,6,7]$ and the references therein.

Now, we are in a position to prove a Ran-Reurings type local fixed point theorem for graphic contractions. First, we need some additional notations.

If $(X, d)$ is a metric space, $x_{0} \in X$ and $r>0$, then we denote

$$
B\left(x_{0}, r\right):=\left\{x \in X: d\left(x_{0}, x\right)<r\right\}, \tilde{B}\left(x_{0}, r\right):=\left\{x \in X: d\left(x_{0}, x\right) \leq r\right\} .
$$

We have the following local fixed point theorem, which generalizes Theorem 2.1, as well as some local results from [4].

Theorem 2.2. Let $X$ be a nonempty set endowed with a partial order relation " $\preceq$ ” and let $d: X \times X \rightarrow$ $\mathbb{R}_{+}$be a complete metric on $X$. Let $x_{0} \in X, r>0$ and $f: B\left(x_{0}, r\right) \rightarrow X$ be an operator which has closed graph with respect to $d$ and is increasing with respect to " $\preceq "$. We suppose:

(i) there exists $\alpha \in] 0,1[$ such that

$$
d\left(f(x), f^{2}(x)\right) \leq \alpha d(x, f(x)), \text { for all } x \in B\left(x_{0}, r\right) \text { with } x \preceq x_{0} ;
$$

(ii) $f\left(x_{0}\right) \preceq x_{0}$;

(iii) $d\left(x_{0}, f\left(x_{0}\right)\right)<(1-\alpha) r$.

Then Fix $(f) \neq \emptyset$ and the sequence of successive approximations $\left(f^{n}\left(x_{0}\right)\right)_{n \in \mathbb{N}}$ converges to a fixed point of $f$. Moreover, if $x^{*}:=\lim _{n \rightarrow \infty} f^{n}\left(x_{0}\right)$, then the following priori estimation holds

$$
d\left(f^{n}\left(x_{0}\right), x^{*}\right) \leq \frac{\alpha^{n}}{1-\alpha} d\left(x_{0}, f\left(x_{0}\right)\right), \text { for each } n \in \mathbb{N} .
$$


Proof. Let $0<s<r$ be such that $d\left(x_{0}, f\left(x_{0}\right)\right) \leq(1-\alpha) s<(1-\alpha) r$. By (ii) and the monotonicity assumption on $f$, we get that

$$
x_{0} \succeq f\left(x_{0}\right) \succeq f^{2}\left(x_{0}\right) \succeq \cdots \succeq f^{n}\left(x_{0}\right) \succeq \cdots .
$$

We can show now that $f^{n}\left(x_{0}\right) \in \tilde{B}\left(x_{0}, s\right)$, for every $n \in \mathbb{N}$. More precisely, we will prove by mathematical induction that

$$
d\left(x_{0}, f^{n}\left(x_{0}\right)\right) \leq\left(1-\alpha^{n}\right) s, \text { for every } n \in \mathbb{N}, n \geq 2 .
$$

Indeed, we have

$$
\begin{aligned}
d\left(x_{0}, f^{2}\left(x_{0}\right)\right) & \leq d\left(x_{0}, f\left(x_{0}\right)\right)+d\left(f\left(x_{0}\right), f^{2}\left(x_{0}\right)\right) \\
& \leq d\left(x_{0}, f\left(x_{0}\right)\right)+\alpha d\left(x_{0}, f\left(x_{0}\right)\right) \\
& \leq(1-\alpha) s+\alpha(1-\alpha) s \\
& =\left(1-\alpha^{2}\right) s .
\end{aligned}
$$

By mathematical induction, we obtain

$$
\begin{aligned}
d\left(x_{0}, f^{n}\left(x_{0}\right)\right) & \leq d\left(x_{0}, f^{n-1}\left(x_{0}\right)\right)+d\left(f^{n-1}\left(x_{0}\right), f^{n}\left(x_{0}\right)\right) \\
& \leq\left(1-\alpha^{n-1}\right) s+\alpha d\left(f^{n-2}\left(x_{0}\right), f^{n-1}\left(x_{0}\right)\right) \\
& \leq \cdots \\
& \leq\left(1-\alpha^{n-1}\right) s+\alpha^{n-1} d\left(x_{0}, f\left(x_{0}\right)\right) \\
& \leq\left(1-\alpha^{n-1}\right) s+\alpha^{n-1}(1-\alpha) s \\
& =\left(1-\alpha^{n}\right) s .
\end{aligned}
$$

Hence $\left.f^{n}\left(x_{0}\right)\right) \in \tilde{B}\left(x_{0}, s\right)$, for every $n \in \mathbb{N}$.

Moreover, by the assumptions (i), (ii) and the monotonicity of $f$, we easily obtain that

$$
d\left(f^{n}\left(x_{0}\right), f^{n+1}\left(x_{0}\right)\right) \leq \alpha^{n} d\left(x_{0}, f\left(x_{0}\right)\right), \text { for each } n \in \mathbb{N} .
$$

Thus, the sequence $\left(f^{n}\left(x_{0}\right)\right)_{n \in \mathbb{N}}$ is Cauchy in $\tilde{B}\left(x_{0}, s\right)$ and it converges to an element $x^{*} \in \tilde{B}\left(x_{0}, s\right)$. Since $f$ has closed graph with respect to $d$, we immediately obtain that $x^{*} \in \operatorname{Fix}(f)$. Moreover, we also have

$$
d\left(f^{n}\left(x_{0}\right), f^{n+p}\left(x_{0}\right)\right) \leq \alpha^{n} \frac{1-\alpha^{p}}{1-\alpha} d\left(x_{0}, f\left(x_{0}\right)\right), \text { for each } n \in \mathbb{N} \text { and } p \in \mathbb{N}^{*} .
$$

Letting $p \rightarrow \infty$, we obtain

$$
d\left(f^{n}\left(x_{0}\right), x^{*}\right) \leq \frac{\alpha^{n}}{1-\alpha} d\left(x_{0}, f\left(x_{0}\right)\right), \text { for each } n \in \mathbb{N} .
$$

A dual result is as following.

Theorem 2.3. Let $X$ be a nonempty set endowed with a partial order relation " $\preceq$ ” and let $d: X \times X \rightarrow$ $\mathbb{R}_{+}$be a complete metric on $X$. Let $x_{0} \in X, r>0$ and $f: B\left(x_{0}, r\right) \rightarrow X$ be an operator which has closed graph with respect to $d$ and is increasing with respect to " $\preceq$ ". We suppose:

(i) there exists $\alpha \in] 0,1[$ such that

$$
d\left(f(x), f^{2}(x)\right) \leq \alpha d(x, f(x)), \text { for all } x \in B\left(x_{0}, r\right) \text { with } x \succeq x_{0} ;
$$

(ii) $x_{0} \preceq f\left(x_{0}\right)$; 
(iii) $d\left(x_{0}, f\left(x_{0}\right)\right)<(1-\alpha) r$.

Then Fix $(f) \neq \emptyset$ and the sequence of successive approximations $\left(f^{n}\left(x_{0}\right)\right)_{n \in \mathbb{N}}$ converges to a fixed point of $f$. Moreover, if $x^{*}:=\lim _{n \rightarrow \infty} f^{n}\left(x_{0}\right)$, then the following priori estimation holds

$$
d\left(f^{n}\left(x_{0}\right), x^{*}\right) \leq \frac{\alpha^{n}}{1-\alpha} d\left(x_{0}, f\left(x_{0}\right)\right), \text { for each } n \in \mathbb{N} .
$$

In the same context, we denote

$$
X_{x_{0}}^{\prec}:=\left\{x \in X: x \preceq x_{0} \text { or } x \succeq x_{0}\right\} .
$$

Another fixed point result for increasing operators is the following theorem.

Theorem 2.4. Let $X$ be a nonempty set endowed with a partial order relation ” $\preceq$ ” and let $d: X \times X \rightarrow$ $\mathbb{R}_{+}$be a complete metric on $X$. Let $x_{0} \in X, r>0$ and $f: B\left(x_{0}, r\right) \rightarrow X$ be an operator which has closed graph with respect to $d$ and is increasing with respect to " $\preceq$ ”. We suppose:

(i) there exists $\alpha \in] 0,1[$ such that

$$
d\left(f(x), f^{2}(x)\right) \leq \alpha d(x, f(x)), \text { for all } x \in B\left(x_{0}, r\right) \cap X_{x_{0}}^{\prec} ;
$$

(ii) $x_{0} \preceq f\left(x_{0}\right)$ or $f\left(x_{0}\right) \preceq x_{0}$;

(iii) $d\left(x_{0}, f\left(x_{0}\right)\right)<(1-\alpha) r$.

Then Fix $(f) \neq \emptyset$ and the sequence of successive approximations $\left(f^{n}\left(x_{0}\right)\right)_{n \in \mathbb{N}}$ converges to a fixed point of $f$. Moreover, if $x^{*}:=\lim _{n \rightarrow \infty} f^{n}\left(x_{0}\right)$, then the following priori estimation holds

$$
d\left(f^{n}\left(x_{0}\right), x^{*}\right) \leq \frac{\alpha^{n}}{1-\alpha} d\left(x_{0}, f\left(x_{0}\right)\right), \text { for each } n \in \mathbb{N} .
$$

Proof. Let $0<s<r$ be such that $d\left(x_{0}, f\left(x_{0}\right)\right) \leq(1-\alpha) s<(1-\alpha) r$. Suppose that $x_{0} \preceq f\left(x_{0}\right)$ or $f\left(x_{0}\right) \preceq x_{0}$. Then, by the monotonicity assumption on $f$, we obtain that

$$
x_{0} \preceq f\left(x_{0}\right) \preceq f^{2}\left(x_{0}\right) \preceq \cdots \preceq f^{n}\left(x_{0}\right) \preceq \cdots
$$

or

$$
x_{0} \succeq f\left(x_{0}\right) \succeq f^{2}\left(x_{0}\right) \succeq \cdots \succeq f^{n}\left(x_{0}\right) \succeq \cdots
$$

Then, as before, we can prove that $f^{n}\left(x_{0}\right) \in \tilde{B}\left(x_{0}, s\right)$, for every $n \in \mathbb{N}$. Indeed, we have

$$
\begin{aligned}
d\left(x_{0}, f^{2}\left(x_{0}\right)\right) & \leq d\left(x_{0}, f\left(x_{0}\right)\right)+d\left(f\left(x_{0}\right), f^{2}\left(x_{0}\right)\right) \\
& \leq d\left(x_{0}, f\left(x_{0}\right)\right)+\alpha d\left(x_{0}, f\left(x_{0}\right)\right) \\
& \leq(1-\alpha) s+\alpha(1-\alpha) s \\
& =\left(1-\alpha^{2}\right) s .
\end{aligned}
$$

By mathematical induction, we obtain

$$
\begin{aligned}
d\left(x_{0}, f^{n}\left(x_{0}\right)\right) & \leq d\left(x_{0}, f^{n-1}\left(x_{0}\right)\right)+d\left(f^{n-1}\left(x_{0}\right), f^{n}\left(x_{0}\right)\right) \\
& \leq\left(1-\alpha^{n-1}\right) s+\alpha d\left(f^{n-2}\left(x_{0}\right), f^{n-1}\left(x_{0}\right)\right) \\
& \leq \cdots \\
& \leq\left(1-\alpha^{n-1}\right) s+\alpha^{n-1} d\left(x_{0}, f\left(x_{0}\right)\right) \\
& \leq\left(1-\alpha^{n-1}\right) s+\alpha^{n-1}(1-\alpha) s \\
& =\left(1-\alpha^{n}\right) s .
\end{aligned}
$$


Hence $\left.f^{n}\left(x_{0}\right)\right) \in \tilde{B}\left(x_{0}, s\right)$, for every $n \in \mathbb{N}$.

Moreover, using the assumptions (i), (ii) and the above relations, we obtain that

$$
d\left(f^{n}\left(x_{0}\right), f^{n+1}\left(x_{0}\right)\right) \leq \alpha^{n} d\left(x_{0}, f\left(x_{0}\right)\right), \text { for each } n \in \mathbb{N} .
$$

Thus, the sequence $\left(f^{n}\left(x_{0}\right)\right)_{n \in \mathbb{N}}$ is Cauchy in $\tilde{B}\left(x_{0}, s\right)$. Hence, the sequence $\left(f^{n}\left(x_{0}\right)\right)_{n \in \mathbb{N}}$ converges to an element $x^{*} \in \tilde{B}\left(x_{0}, s\right)$. Since $f$ has closed graph with respect to $d$ we immediately obtain that $x^{*} \in F i x(f)$. The rest of the proof is similar to our previous theorems.

Theorem 2.5. Let $X$ be a nonempty set endowed with a partial order relation " $\preceq$ ” and let $d: X \times X \rightarrow$ $\mathbb{R}_{+}$be a complete metric on $X$. Let $x_{0} \in X, r>0$ and $f: B\left(x_{0}, r\right) \rightarrow X$ be an operator which has closed graph with respect to $d$. We suppose:

(i) there exists $\alpha \in] 0,1[$ such that

$$
d\left(f(x), f^{2}(x)\right) \leq \alpha d(x, f(x)), \text { for all } x \in B\left(x_{0}, r\right) \cap X_{x_{0}}^{\prec} ;
$$

(ii) $x_{0} \preceq f\left(x_{0}\right)$ or $f\left(x_{0}\right) \preceq x_{0}$;

(iii) $d\left(x_{0}, f\left(x_{0}\right)\right)<(1-\alpha) r$;

(iv) $X_{\bar{x}_{0}}^{\prec} \in I(f)$.

Then Fix $(f) \neq \emptyset$ and the sequence of successive approximations $\left(f^{n}\left(x_{0}\right)\right)_{n \in \mathbb{N}}$ converges to a fixed point of $f$. Moreover, if $x^{*}:=\lim _{n \rightarrow \infty} f^{n}\left(x_{0}\right)$, then the following priori estimation holds

$$
d\left(f^{n}\left(x_{0}\right), x^{*}\right) \leq \frac{\alpha^{n}}{1-\alpha} d\left(x_{0}, f\left(x_{0}\right)\right), \text { for each } n \in \mathbb{N} .
$$

Proof. Let $0<s<r$ be such that $d\left(x_{0}, f\left(x_{0}\right)\right) \leq(1-\alpha) s<(1-\alpha) r$. Suppose that $x_{0} \preceq f\left(x_{0}\right)$ or $f\left(x_{0}\right) \preceq x_{0}$. Then, by (iv), we obtain that $\left.f^{n}\left(x_{0}\right)\right) \in X_{\bar{x}_{0}}$, for every $n \in \mathbb{N}^{*}$. Next, we will prove that $f^{n}\left(x_{0}\right) \in \tilde{B}\left(x_{0}, s\right)$, for every $n \in \mathbb{N}$. More precisely, we will prove by mathematical induction that

$$
d\left(x_{0}, f^{n}\left(x_{0}\right)\right) \leq\left(1-\alpha^{n}\right) s, \text { for every } n \in \mathbb{N}, n \geq 2 .
$$

Indeed, we have

$$
\begin{aligned}
d\left(x_{0}, f^{2}\left(x_{0}\right)\right) & \leq d\left(x_{0}, f\left(x_{0}\right)\right)+d\left(f\left(x_{0}\right), f^{2}\left(x_{0}\right)\right) \\
& \leq d\left(x_{0}, f\left(x_{0}\right)\right)+\alpha d\left(x_{0}, f\left(x_{0}\right)\right) \\
& \leq(1-\alpha) s+\alpha(1-\alpha) s \\
& =\left(1-\alpha^{2}\right) s .
\end{aligned}
$$

By mathematical induction, we obtain

$$
\begin{aligned}
d\left(x_{0}, f^{n}\left(x_{0}\right)\right) & \leq d\left(x_{0}, f^{n-1}\left(x_{0}\right)\right)+d\left(f^{n-1}\left(x_{0}\right), f^{n}\left(x_{0}\right)\right) \\
& \leq\left(1-\alpha^{n-1}\right) s+\alpha d\left(f^{n-2}\left(x_{0}\right), f^{n-1}\left(x_{0}\right)\right) \\
& \leq \cdots \\
& \leq\left(1-\alpha^{n-1}\right) s+\alpha^{n-1} d\left(x_{0}, f\left(x_{0}\right)\right) \\
& \leq\left(1-\alpha^{n-1}\right) s+\alpha^{n-1}(1-\alpha) s \\
& =\left(1-\alpha^{n}\right) s .
\end{aligned}
$$

Hence $\left.f^{n}\left(x_{0}\right)\right) \in \tilde{B}\left(x_{0}, s\right)$, for every $n \in \mathbb{N}$. 
Moreover, using the assumptions (i), (ii) and the above relations, we obtain that

$$
d\left(f^{n}\left(x_{0}\right), f^{n+1}\left(x_{0}\right)\right) \leq \alpha^{n} d\left(x_{0}, f\left(x_{0}\right)\right), \text { for each } n \in \mathbb{N} .
$$

Thus, $\left(f^{n}\left(x_{0}\right)\right)_{n \in \mathbb{N}}$ is Cauchy in $\tilde{B}\left(x_{0}, s\right)$. Hence, $\left(f^{n}\left(x_{0}\right)\right)_{n \in \mathbb{N}}$ converges to an element $x^{*} \in \tilde{B}\left(x_{0}, s\right)$. Since $f$ has closed graph with respect to $d$, we immediately obtain that $x^{*} \in F i x(f)$. The rest of the proof follows in a similar way to our previous results.

Remark 2.1. It is easy to see that the assumption (i) in Theorem 2.4 or Theorem 2.5 implies the assumption (i) in Theorem 2.2 or Theorem 2.3. Notice that the reverse implication does not hold.

It is an open question to obtain the convergence (to a fixed point of $f$ ) of the sequence $\left(f^{n}(x)\right)_{n \in \mathbb{N}}$ of successive approximations of $f$ starting from an arbitrary point $x \in X$ with $x \preceq x_{0}$ or $x \succeq x_{0}$ or, more generally, from any $x \in X$. It this last case, the operator $f$ is a weakly Picard operator. Another question is when a local graphic contraction is a weakly $\psi$-Picard operator.

A partial answer is given in the next result. For this theorem, we need the concept of ordered metric space.

Definition 2.2. Let $X$ be a nonempty set. A triple $(X, d, \preceq)$ is called an ordered metric space if:

(a) $(X, d)$ is a metric space;

(b) $(X, \preceq)$ is a partially ordered set;

(c) if $\left(u_{n}\right)_{n \in \mathbb{N}}$ and $\left(v_{n}\right)_{n \in \mathbb{N}}$ are sequences in $X$ such that $u_{n} \preceq v_{n}$ for every $n \in \mathbb{N}$ and $u_{n} \rightarrow u, v_{n} \rightarrow v$ as $n \rightarrow \infty$, then $u \preceq v$.

Theorem 2.6. Let $(X, d, \preceq)$ be an ordered metric space, where $d: X \times X \rightarrow \mathbb{R}_{+}$is a complete metric on $X$. Let $x_{0} \in X$ and let $f: X \rightarrow X$ be an operator which has closed graph with respect to $d$. We suppose:

(i) there exists $\alpha \in] 0,1[$ such that

$$
d\left(f(x), f^{2}(x)\right) \leq \alpha d(x, f(x)), \text { for all } x \in X_{x_{0}}^{\prec} ;
$$

(ii) $X_{x_{0}}^{\prec} \in I(f)$.

Then Fix $(f) \neq \emptyset$ and, for every $x \in X_{\bar{x}_{0}}$, the sequence $\left(f^{n}(x)\right)_{n \in \mathbb{N}}$ of successive approximations of $f$ converges to a fixed point of $f$. Moreover, if, for $x \in X_{x_{0}}^{\prec}$, we denote $x^{*}(x):=\lim _{n \rightarrow \infty} f^{n}(x)$, then the following priori estimation holds

$$
d\left(f^{n}(x), x^{*}(x)\right) \leq \frac{\alpha^{n}}{1-\alpha} d(x, f(x)), \text { for every } n \in \mathbb{N} \text { and each } x \in X_{x_{0}}^{\prec} .
$$

Proof. Notice first that, since $(X, d, \preceq)$ is an ordered metric space, $X_{\bar{x}_{0}}$ is closed in $(X, d)$. Hence, $f: X_{\bar{x}_{0}} \rightarrow X_{\bar{x}_{0}}^{\preceq}$ is a graphic contraction with closed graph. Thus, the conclusion follows by the above mentioned fixed point theorem of Rus, see [11] or the graphic contraction principle in [14].

For related results and applications of the graphic contraction theory, see $[8,9,15]$ and the references therein.

Remark 2.2. It is an open question to extend the above results to the multi-valued case. 


\section{REFERENCES}

[1] V. Berinde, A. Petruşel, I.A. Rus, M.-A. Şerban, The retraction-displacement condition in the theory of fixed point equation with a convergent iterative algorithm, In: T. Rassias, V. Gupta (Eds.) Mathematical Analysis, Approximation Theory and Their Applications, Springer Optimization and Its Applications, vol. 111, pp. 75-106, Springer, Cham, 2016.

[2] P. Chaoha, W. Sudprakhon, Fixed point sets of Subrahmanyam maps, Linear Nonlinear Anal. 3 (2017), 149-154.

[3] T.L. Hicks, B.E. Rhoades, A Banach type fixed point theorem, Math. Japonica 24 (1979), 327-330.

[4] T.A. Lazăr, A. Petruşel, N. Shahzad, Fixed points for non-self operators and domain invariance theorems, Nonlinear Anal. 70 (2009), 117-125.

[5] J.J. Nieto, R.L. Pouso, R. Rodríguez-López, Fixed point theorems in ordered abstract spaces, Proc. Amer. Math. Soc. 135 (2007), 2505-2517.

[6] J.J. Nieto, R. Rodríguez-López, Existence and uniqueness of fixed point in partially ordered sets and applications to ordinary differential equations, Acta Math. Sinica, Engl. Ser. 23 (2007), 2205-2212.

[7] A. Petruşel, I.A. Rus, Fixed point theorems in ordered L-spaces, Proc. Amer. Math. Soc. 134 (2005), 411-418.

[8] A. Petruşel, I.A. Rus, M.-A. Şerban, Nonexpansive operators as graphic contractions, J. Nonlinear Convex Anal. 17 (2016), 1409-1415.

[9] A. Petruşel, I.A. Rus, Graphic Contractions and Applications, Mathematical Analysis and Applications, Springer, 2018.

[10] A.C.M. Ran, M.C.B. Reurings, A fixed point theorem in partially ordered sets and some applications to matrix equations, Proc. Amer. Math. Soc. 132 (2004), 1435-1443.

[11] I.A. Rus, The method of successive approximations, Rev. Roum. Math. Pure Appl. 17 (1972), 1433-1437.

[12] I.A. Rus, Picard operators and applications, Sci. Math. Japonicae 58 (2003), 191-219.

[13] I.A. Rus, Relevant classes of weakly Picard operators, Ann. Univ. de Vest Timisoara Seria Math. Inform. 2 (2016), 131147.

[14] I.A. Rus, A. Petruşel, G. Petruşel, Fixed Point Theory, Cluj University Press, Cluj-Napoca, 2008.

[15] M.-H. Shih, W. Takahashi, Positive stochastic matrices as contractions, J. Nonlinear Convex Anal. 14 (2013), 649-650.

[16] P.V. Subrahmanyam, Remarks on some fixed point theorems related to Banach's contraction principle, J. Math. Phys. Sci. 8 (1974), 445-457. 